\title{
Structure of the XPA DNA binding domain and RPA high affinity DNA binding domains on a model NER substrate
}

\author{
Walter J. Chazin ${ }^{a}$, Agnieszka M. Topolska-Wośa , Norie Sugitani ${ }^{a}$, John J. \\ Cordoba $^{a}$, Hyun Suk Kim ${ }^{b}$, Jung-Eun $\mathrm{Yeo}^{b}$, Daniel Rosenberg ${ }^{\mathrm{c}}$, Michal Hammel ${ }^{\mathrm{c}}$ \\ and Orlando D. Schärer ${ }^{\mathrm{b}}$ \\ ${ }^{a}$ Departments of Biochemistry and Chemistry, and Center for Structural Biology, \\ Vanderbilt University, Nashville, TN 37240-7917, USA, walter.chazin@vanderbilt.edu \\ ${ }^{\mathrm{b}}$ Center for Genomic Integrity, Institute for Basic Science, Ulsan, Republic of Korea \\ ${ }^{\mathrm{C}}$ Division of Molecular Biophysics and Integrated Bioimaging, Lawrence Berkeley \\ National Laboratory, Berkeley, CA 94720, USA
}

The nucleotide excision repair (NER) pathway is responsible for repairing the majority of bulky DNA lesions in the genome. The range of lesions repaired by NER is large, spanning from cross-links arising from sunlight and cancer therapies to large chemical adducts to DNA from environmental toxins and car exhaust. Defects in human NER result in the genetic disorder xeroderma pigmentosum (XP), which is associated with extreme sensitivity to sunlight and a high disposition to skin cancer and neurodegeneration.

Repair of damaged DNA by NER occurs via the progressive processing of the substrate by a multi-protein machine composed of more than 20 proteins. At the core of this machinery is a central scaffold generated by the coordinated action of the $X P$ complementation group A protein (XPA) together with the single stranded DNA (ssDNA) binding protein replication protein A (RPA). This scaffold serves to properly orient NER factors on the damaged DNA substrate and position them within the multi-protein NER machinery. Despite their central role in NER, XPA-RPA interactions are not well understood. In fact, even their positioning on the substrate is not yet established.

XPA interacts with RPA through a primary contact involving the XPA N-terminus and the RPA32C protein recruitment domain and a second weaker interaction between the XPA DNA binding domain (DBD) and the RPA tandem high affinity ssDNA binding domains (RPA70AB). We have use a hybrid structural approach to determine the structure of the complex of XPA DBD and RPA bound to a model NER substrate. We started from X-ray crystal structures of yeast XPA (Rad14) DBD bound to a damaged DNA duplex and RPA70AB bound to $\mathrm{dC}_{8}$. Reciprocal NMR titrations were used to map the interfaces between the two proteins. These data revealed XPA DBD binds ssDNA on the same face as RPA70AB, but that their binding sites are independent. Data from NMR and SAXS were then fed into two independent docking programs and the resulting structures were analyzed and refined.

Based on the structural information, mutations in the XPA DBD at its RPA70AB interface were designed and validated, supporting the structural model and providing key reagents for functional analysis. The mutant XPA proteins were found to exhibit decreased NER activity in cell extracts. These results demonstrate the functional importance of this contact point between XPA and RPA, despite the relatively weak affinity. Our results inform on the controversy about where XPA is bound on NER substrates, provide novel insights to help predict the molecular basis of diseaseassociated XPA missense mutations, and contribute to the growing understanding of the structure and mechanical action of the NER machinery. 